\title{
A Retrospective Study for Detection of Inducible Resistance to Clindamycin in Staphylococcus aureus Isolates Using D-Test at RIMS Teaching Hospital, Raichur, India
}

\author{
R. Venkatesh Naik*, Basavaraj V. Peerapur and P. Sandhya \\ Department of Microbiology, RIMS, Raichur, Karnataka, India \\ *Corresponding author
}

\begin{tabular}{|c|}
\hline Keywords \\
\hline $\begin{array}{l}\text { Clindamycin } \\
\text { resistance, MRSA, } \\
\text { MSSA, D test. }\end{array}$ \\
\hline Article Info \\
\hline $\begin{array}{l}\text { Accepted: } \\
\text { 14 September } 2017 \\
\text { Available Online: } \\
10 \text { October } 2017\end{array}$ \\
\hline
\end{tabular}

\section{Keywords}

Clindamycin resistance, MRSA MSSA, D test. 10 October 2017

\section{A B S T R A C T}

Staphylococcus aureus is recognized as causing nosocomial and community-acquired infections in every region of the world. Clindamycin is considered as useful alternate drug in penicillin-allergic patients in the treatment of skin \& soft tissue infections caused by Staphylococcus aureus. Staphylococcus spp. can be resistant to erythromycin through either erm or msr A genes. Strains with erm-mediated erythromycin resistance may possess inducible Clindamycin resistance but may appear susceptible to Clindamycin by disc diffusion test. The objective of the present study was to know the prevalence of erythromycin-induced Clindamycin resistance among clinical isolates of $S$. aureus. A total of $160 \mathrm{~S}$. aureus isolates from various clinical samples submitted in the Dept. of Microbiology, RIMS teaching hospital, Raichur were studied. Methicillin resistant $S$. aureus strains were identified by Cefoxitin disc diffusion method. Inducible Clindamycin resistance was detected by erythromycin and Clindamycin disc approximation test (D-zone test) as per CLSI guidelines. Among the $160 \mathrm{~S}$. aureus isolates, 75 strains (46.8\%) were detected as MRSA of which 32(42.6\%) strains showed inducible Clindamycin resistance (D-test positive) and 12(14.1\%) isolates out of 85 strains MSSA were D-test positive. $73(45.6 \%)$ isolates of $S$. aureus were sensitive to both erythromycin and Clindamycin. High prevalence of strains with inducible Clindamycin resistance particularly among MRSA indicates that inducible Clindamycin resistance testing (D-test) should be included as a part of routine antibiotic susceptibility. These isolates may be missed in routine antibiotic testing by disk diffusion method.

\section{Introduction}

Staphylococci are important causative agents of nosocomial and community acquired infections in skin and soft tissues. Emergence of methicillin resistance in Staphylococci has left us with very few therapeutic alternatives available to treat. The Macrolide lincosamide Streptogramin B (MLSB) family of antibiotics serves as one such alternative with Clindamycin being preferred agent due to its excellent pharmacokinetic properties. ${ }^{[1]}$
Strains with inducible resistance to Clindamycin are difficult to detect in the routine laboratory test, as they appear erythromycin resistant and Clindamycin sensitive in vitro, when not placed adjacent to each other. In such cases, in-vivo therapy with Clindamycin may select therapeutic failure. ${ }^{[2,}$ 3] However widespread use of Macrolideslincosamides-Streptogramin B (MLSB) antibiotics has led to an increased resistance 
to these antibiotics by Staphylococcal strains. ${ }^{[4]}$

The Macrolide-Lincosamide-Streptogramin B (MLSB) family of antibiotics is commonly used in the treatment of Staphylococcal infections. Clindamycin a protein synthesis inhibitor is an attractive option for use in the scenario of increasing drug resistance among the Staphylococci especially for skin and soft tissue infections and as an alternative in Penicillin allergic patients.

This drug has excellent tissue penetration, requires no renal dosing adjustments and has a good oral absorption. All these factors make it convenient for outpatient prescription or as follow-up after intravenous therapy. ${ }^{[5]}$ However, one important issue with Clindamycin treatment is the risk of clinical failure during therapy.

The MLS family of antibiotics has three different mechanisms of resistance: target site modification, enzyme antibiotic inactivation and Macrolide efflux pumps. ${ }^{[6]}$ Macrolide antibiotic resistance in Staphylococcus aureus and Coagulase negative Staphylococci (CONS) may be due to an active efflux mechanism encoded by msr A (macrolides Streptogramin resistance) genes, conferring resistance to macrolides and type B Streptogramin only ${ }^{[7]}$ or may be due to ribosomal target modification, affecting macrolides, lincosamides, and type B Streptogramin (MLSB resistance). Erm (erythromycin resistant methylene) genes are responsible for encoding enzymes that confer inducible or constitutive resistance to MLS agents via methylation of the 23S rRNA and reducing binding by MLS agents to the ribosome. ${ }^{[8]}$ Antimicrobial susceptibility data are important for the management of infections, but false susceptibility results may be obtained if isolates are not tested for inducible Clindamycin resistance. This resistance missed by using standard susceptibility test methods such as standard broth-based or agar dilution susceptibility tests. The inducible MLSB resistance can be detected by a simple test known as Disk approximation test or D test. Low level of Erythromycin is an inducer of MLSBi phenotype and this is the basis of performing D test. ${ }^{[9]}$ Failure to identify inducible MLSB resistance may lead to clinical failure of Clindamycin therapy. Conversely, labelling all erythromycin-resistant staphylococci as Clindamycin resistant prevents the use of Clindamycin in infections caused by truly Clindamycin-susceptible Staphylococcal isolates.

The present study was conducted for detection of inducible resistance to Clindamycin in Staphylococcus aureus isolates using D - test at Raichur Institute of Medical Sciences-teaching hospital, RAICHUR, Karnataka, India.

\section{Materials and Methods}

The study was conducted from September 2014 to April 2016 in the Department of Microbiology, Raichur Institute of Medical Sciences-teaching hospital, RAICHUR, Karnataka, India. A total of 160 S. aureus strains were isolated from various clinical specimens like pus, wound swabs, aspirates, blood, sterile fluids etc. Only one isolate per patient was included in the study. All the isolates were tested for their susceptibility to penicillin (10 units), Gentamicin (10 $\mu \mathrm{g})$, tetracycline $(30 \mu \mathrm{g})$, Cotrimoxazole $(25 \mu \mathrm{g})$, erythromycin $(15 \mu \mathrm{g})$, ciprofloxacin $(5 \mu \mathrm{g})$, Vancomycin $(30 \mu \mathrm{g})$ and linezolid $(30 \mu \mathrm{g})$ by Kirby Bauer disc diffusion method using criteria of standard zone of inhibition. Methicillin resistance was detected by Cefoxitin disk diffusion method using a $30 \mu \mathrm{g}$ disk (Hi-media laboratories Pvt. Ltd., Mumbai). 


\section{D-test}

D-test Negative (MS Phenotype): Isolates showing resistance to Erythromycin ( $\leq$ $13 \mathrm{~mm}$ ) but susceptible to Clindamycin ( $\geq$ $21 \mathrm{~mm}$ ) and showing circular zone of inhibition around Clindamycin.

Constitutive Resistance (cMLSB Phenotype): Isolates showing resistance to both Erythromycin $(\leq 13 \mathrm{~mm})$ and Clindamycin $(\leq$ $14 \mathrm{~mm}$ ) with circular zone of inhibition if any around Clindamycin.

D-test Positive (iMLSB Phenotype): Isolates showing resistance to Erythromycin ( $\leq$ $13 \mathrm{~mm})$ and sensitive to Clindamycin $(\geq$ $21 \mathrm{~mm}$ ) and showing $\mathrm{D}$ shaped zone of inhibition around Clindamycin with the flattening towards Erythromycin (Fig. 1).

It was observed that many of the isolates were showing resistance to Cefoxitin $(\leq 19 \mathrm{~mm})$ on routine testing. Suspecting them as MRSA producers, these isolates were further tested for oxacillin $(1 \mu \mathrm{g})$ resistance or susceptibility pattern. The results were tabulated and analysed.

\section{Statistical analysis}

The data were entered and analysed using Statistical Package for Social Sciences SPSS software version21.0. P - Value $<0.05$ was considered statistically significant.

\section{Results and Discussion}

Among the 160 S. aureus strains studied, 44 (27.5\%) strains were D-test positive i.e. of the inducible MLSB (iMLSB) phenotype as compared to the $25(15.6 \%)$ constitutive MLSB (cMLSB) phenotypic strains (Table 1). High percentage of erythromycin resistance 87 (54.3\%) was noted among S. aureus strains. In our study, 75 strains $(46.8 \%)$ were detected as MRSA of which 32 (42.6\%) strains showed inducible Clindamycin resistance. Percentage of both inducible and constitutive resistance was found to be higher amongst MRSA isolates as compared to MSSA $(p<0.001)$ (Table 1).

Clindamycin, a lincosamide, is one of the most efficient antibiotics in treating staphylococcal skin and soft tissue infections, including osteomyelitis because of its excellent tissue penetration except in CNS. ${ }^{[10]}$ It accumulates in abscesses and no dosage requirements are needed in the presence of renal disease. It also directly inhibits the staphylococcal toxin production and is a useful alternative for patients who are allergic to penicillin. ${ }^{[11]}$

Clindamycin resistance can develop in staphylococcal isolates with inducible phenotype, and such isolates, can undergo a rapid in vitro and in vivo conversion to a constitutive resistance phenotype. ${ }^{[12]}$

Reporting $S$. aureus as susceptible to Clindamycin without checking for inducible resistance may result in institution of inappropriate Clindamycin therapy. On the other hand negative result for inducible Clindamycin resistance confirms Clindamycin susceptibility and provides a very good therapeutic option. ${ }^{[13]}$ Therefore accurate susceptibility data are important for proper treatment decisions. This is where the D-test becomes significant.

In present study, when $S$. aureus isolates were subjected to D-zone test, it was found that 44 $(27.5 \%)$ isolates showed inducible Clindamycin resistance (iMLSB phenotype) and $25 \quad(15.6 \%)$ showed constitutive resistance (cMLSB phenotype). A study from Bangalore reported that $24.9 \%$ of their $S$. aureus strains were of iMLSB phenotype and $18.3 \%$ were of cMLSB phenotype. ${ }^{[14]}$ 
There have been various reports on the pattern of the MLSB resistance among the staphylococci; some reports indicate a high prevalence of the iMLSB phenotype, while the others indicate an increasing frequency of the cMLSB phenotype. The true incidence depends on the patient population studied, the geographical region, the hospital characteristics and methicillin susceptibility. ${ }^{[10]}$ In this study, it was found that both the inducible and constitutive Clindamycin resistance were seen in significantly higher proportion among MRSA as compared to MSSA isolates $(p<0.001)$. Studies from different parts of India have reported $30 \%$ to $64 \%$ of the MRSA isolates to be of the iMLSB phenotype. 14 In the present study, 32 (42.6\%) of the 75 MRSA isolates were found to be of iMLSB phenotype which is higher than the findings of Deotale et al., who reported $27.6 \%$ iMLSB resistance in the MRSA isolates. ${ }^{[13]}$

Table.1 Comparison of different types of MLSB resistance among S. aureus on D-test

\begin{tabular}{|c|c|c|c|}
\hline $\begin{array}{c}\text { PHENOTYPE } \\
\text { (SUSCEPTIBILITY PATTERN) }\end{array}$ & $\begin{array}{c}\text { MRSA (\%) } \\
(\mathrm{n}=75)\end{array}$ & $\begin{array}{c}\text { MSSA (\%) } \\
(\mathrm{n}=\mathbf{8 5})\end{array}$ & $\begin{array}{c}\text { TOTAL }(\%) \\
(\mathrm{n}=160)\end{array}$ \\
\hline $\begin{array}{c}\text { Inducible Clindamycin resistance } \\
\text { (ER-R, CL-S, D test + ve) }\end{array}$ & $\mathbf{3 2}(\mathbf{4 2 . 6 \% )}$ & $\mathbf{1 2}(\mathbf{1 4 . 1 \% )})$ & $\mathbf{4 4}(\mathbf{2 7 . 5 \% )})$ \\
\hline $\begin{array}{c}\text { Constitutive Clindamycin } \\
\text { resistance (ER-R, CL-R) }\end{array}$ & $\mathbf{1 8}(\mathbf{2 4 . 0 \% )}$ & $\mathbf{7 ( 8 . 2 \% )}$ & $\mathbf{2 5}(\mathbf{1 5 . 6 \% )})$ \\
\hline $\begin{array}{c}\text { MS Phenotype } \\
\text { (ER-R, CL-S, D test -ve) }\end{array}$ & $\mathbf{1 0}(\mathbf{1 3 . 3 \%})$ & $\mathbf{8}(\mathbf{9 . 4 \%})$ & $\mathbf{1 8}(\mathbf{1 1 . 2 \% )})$ \\
\hline $\begin{array}{c}\text { Susceptible to Erythromycin \& } \\
\text { Clindamycin (ER-S, CL-S) }\end{array}$ & $\mathbf{1 5}(\mathbf{2 0 . 0 \% )}$ & $\mathbf{5 8}(\mathbf{6 8 . 2 \%})$ & $\mathbf{7 3 ( 4 5 . 6 \% )}$ \\
\hline
\end{tabular}

Fig.1

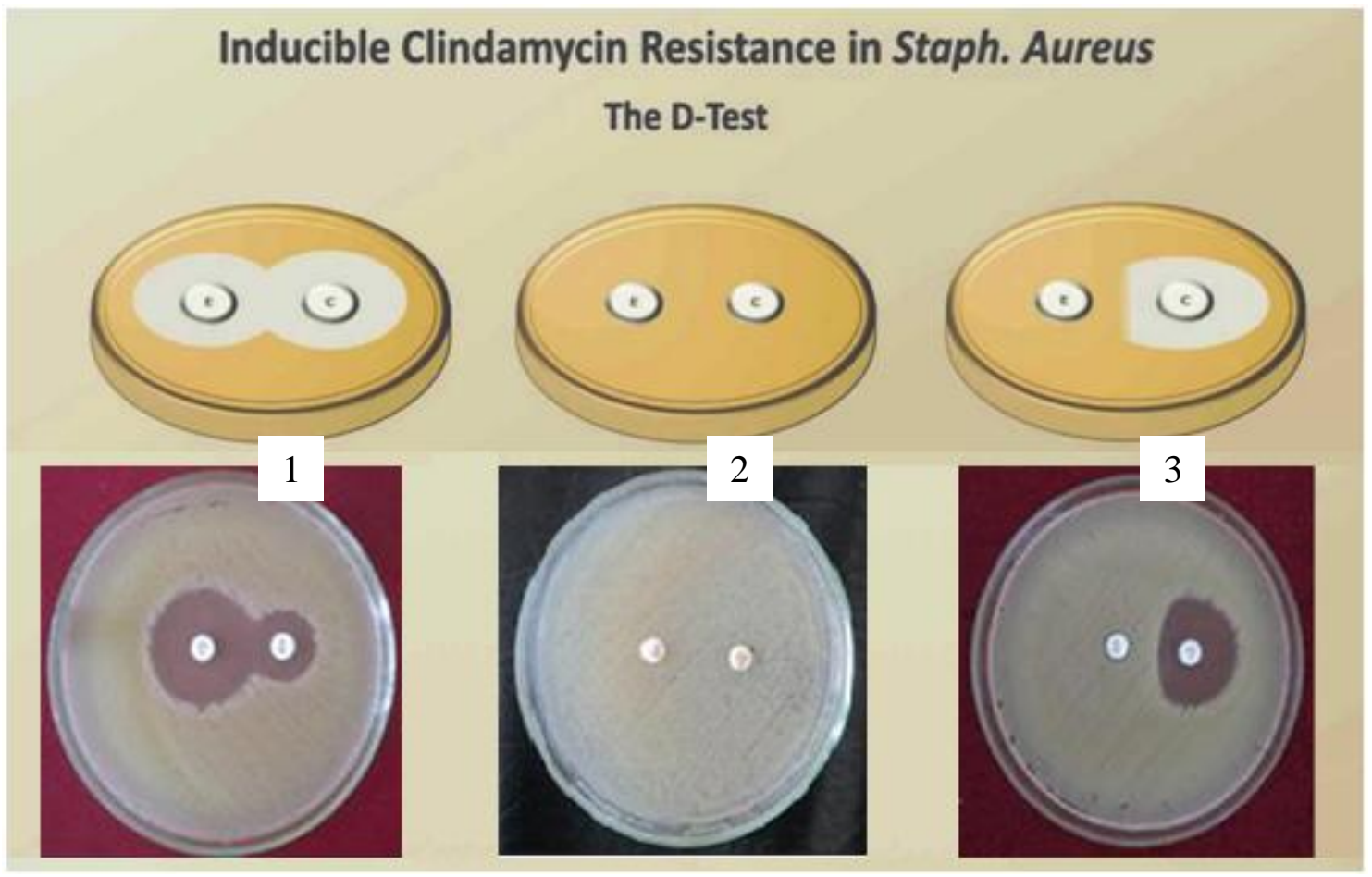


In this study, $13.3 \%$ MRSA belonged to MS phenotype as compared to $9.4 \%$ MSSA. Similar findings were made by Gadepalli et al., reported $12.0 \%$ strains of the MS phenotype among the MRSA and MSSA each.

In present study, $46.8 \%$ of the total isolates of the $S$. aureus were MRSA. Other studies have also shown such a high prevalence of MRSA from various parts of the country ranging from $31 \%$ to $44 \% .^{[15,16]}$ Lack of awareness, the indiscriminate and improper use of antibiotics before coming to the hospital might be the contributory factors for such a high prevalence of MRSA. Even though there are recent reports of the increase in emergence of Vancomycin resistance of S.aureus worldwide. ${ }^{[17,18]}$

As Clindamycin is one of the most commonly used antibiotics for MRSA isolates, the increasing Clindamycin resistance in the form of iMLSB and cMLSB limits the therapeutic options for MRSA to the antibiotics like linezolid and Vancomycin.

The inducible Clindamycin resistance can be easily missed by routine in vitro susceptibility tests, when the erythromycin and the Clindamycin discs are placed in non-adjacent positions. In view of the therapeutic implications, the D test is a simple, reliable and inexpensive test to perform along with routine susceptibility testing which delineates the inducible (iMLSB) and the constitutive (cMLSB) resistance.

The incidence of resistance is highly variable with regard to geographic locality; hence the local data regarding inducible Clindamycin resistance is helpful in guiding antistaphylococcal therapy. Use of D test in a routine laboratory will enable us in guiding the clinicians regarding the judicious use of Clindamycin.

\section{References}

1. Fiebelkorn KR, Crawford SA, McElmeel ML, Jorgenson JH. Practical disc diffusion method for detection of inducible Clindamycin resistance in Staphylococcus aureus and coagulase negative Staphylococci. J Clin Microbiol, 2003; 41:4740-4.

2. Steward CD, Raney PM, Morrell AK, Williams PP, McDougal LK, Jevitt, et al. Testing for induction of Clindamycin resistance in erythromycin resistant isolates of Staphylococcus aureus. J Clin Microbiol, 2005; 43:1716-21.

3. Deotale V, Mendiratta DK, Rant U, Narang P. Inducible Clindamycin resistance in Staphylococcus aureus isolated from clinical samples. Indian $\mathbf{J}$ Med Microbiol, 2010; 28:124-126.

4. Gadepalli R, Dhawan B, Mohanty S, Kapil A, Das BK, Choudhary R. Inducible Clindamycin resistance in clinical isolates of Staphylococcus aureus. Indian J med Res, 2006; 123: 571-3.

5. Drinkovic D, Fuller E R, Shore KP, Holland DJ, Ellis -Pegler R. Clindamycin treatment of Staphylococcus aureus expressing inducible Clindamycin resistance. J Antimicrob Chemother, 2001; 48: 315-316.

6. Yalmaz G, Aydin K, Iskender S, Caylan $\mathrm{R}$, Koksal I. Detection and prevalence of inducible Clindamycin resistance in Staphylococci. J Med Microbiol, 2007; 56 (Pt 3): 342-5.

7. Ross J I, Eady E A, Cove J H, Cunliffe W J, Baumberg S, Wootton J C. Inducible erythromycin resistance in staphylococci is encoded by a member of the ATPbinding transport super-gene family. Mol Microbiol, 1990; 4: 1207-1214.

8. Roberts MC, Sutcliffe J, Courvalin P, Jensen LB, Rood J, Seppala H. Nomenclature for Macrolide-lincosamideStreptogramin resistance determinants. 
Antimicrob Agents Chemother, 1999; 43:2823-2830.

9. Ciraj A M, Vinod P, Sreejith G, Rajani K. Inducible Clindamycin resistance among clinical isolates of Staphylococci. Ind $\mathrm{J}$ Pathol Microbiol, 2009; 52(1):49-51.

10. Mohanasoundaram KM. The prevalence of inducible Clindamycin resistance among gram positive cocci from various clinical specimens. Journal of Clinical and Diagnostic Research, 2011; 5:38-40.

11. Kasten MJ. Clindamycin, metronidazole, and chloramphenicol. Mayo Clin Proc, 1999; 74:825-33

12. Yilmaz G, Aydin K, Iskender S, Caylan R, Koksal I. Detection and prevalence of inducible Clindamycin resistance in staphylococci. J Med Microbiol, 2007; 56:342-5.

13. Deotale V, Mendiratta DK, Raut U, Narang P. Inducible Clindamycin resistance in Staphylococcus aureus isolated from clinical samples. Indian $\mathbf{J}$ Med Microbiol, 2010; 28:124-6.

14. Shantala GB, Shetty AS, Rao RK, Vasudeva, Nagarathnamma T. Detection of inducible Clindamycin resistance in clinical isolates of Staphylococcus aureus by the disc diffusion induction test. Journal of Clinical and Diagnostic Research, 2011; 5:35-7.

15. Anbumani N, Kalyani J, Mallika M. Prevalence of methicillin-resistant Staphylococcus aureus in a Tertiary Referral Hospital in Chennai, South India. Indian Journal for the Practising Doctor, 2006-08 - 2006-09; 3(4).

16. Tyagi A, Kapil A, Singh P. Incidence of methicillin resistant Staphylococcus aureus (MRSA) in pus samples at a tertiary care hospital, AIIMS, New Delhi. Journal Indian Academy of Clinical Medicine, 2008; 9(1): 33-5.

17. Fridkin SK. Vancomycin-intermediate and resistant Staphylococcus aureus: what the infectious disease specialist needs to know. Clin Infect Dis, 2001; 32:108-15.

18. Bal M, Saha B, Singh AK, Ghosh A. Identification and characterization of a vancomycinresistant Staphylococcus aureus isolated from Kolkata (South Asia). J Med Microbiol, 2008; 57:172-79.

\section{How to cite this article:}

Venkatesh Naik R., Basavaraj V. Peerapur and Sandhya P. 2017. A Retrospective Study for Detection of Inducible Resistance to Clindamycin in Staphylococcus aureus Isolates Using D Test at RIMS Teaching Hospital, Raichur. Int.J.Curr.Microbiol.App.Sci. 6(10): 1537-1542. doi: https://doi.org/10.20546/ijcmas.2017.610.xx 